\title{
Accelerating design and transforming baccalaureate nursing education to foster a culture of health
}

\author{
Mary Theresa Bouchaud ${ }^{* 1}$, Beth Ann Swan ${ }^{1}$, Angela M. Gerolamo ${ }^{1}$, Kathleen Black ${ }^{1}$, Karen Alexander ${ }^{1}$, Jennifer \\ Bellot $^{1}$, Kathryn Shaffer ${ }^{1}$, Catherine Levonian ${ }^{1}$, Denise Brown ${ }^{1}$, Susan Egger ${ }^{1}$, Rachel Scherzer ${ }^{1}$, Dori Taylor Sullivan ${ }^{2}$ \\ ${ }^{1}$ Jefferson College of Nursing, Thomas Jefferson University, Philadelphia, Pennsylvania, United States \\ ${ }^{2}$ Leadership, Education, Quality Consulting, Stuart, Florida, United States
}

Received: November 16, 2015

Accepted: June 27, 2016

Online Published: July 6, 2016

DOI: $10.5430 /$ jnep.v6n11p97

URL: http://dx.doi.org/10.5430/jnep.v6n11p97

\begin{abstract}
Healthcare reform and changing population health demographics call for a radical transformation in healthcare delivery and the education of healthcare providers. Nurses comprise the largest proportion of healthcare providers making it necessary to ensure that they are prepared to address the challenges that arise from the evolving healthcare delivery system. A key message of The Institute of Medicine's The Future of Nursing: Leading Change, Advancing Health, is that nurses must lead healthcare change. To accomplish this, nurses must recognize their role in educating the new nursing workforce about creating a culture of health. Specifically, nurse educators must act as stewards for promoting health and wellness, and reducing health disparities and inequities. They must also recognize their role in forming partnerships with community organizations to improve primary care and population health by addressing social determinants of health. The purpose of this paper is to describe the structure for developing an innovative baccalaureate nursing curriculum and lessons learned that can inform the efforts of others interested in accelerating design of new curriculum.
\end{abstract}

Key Words: Curriculum innovation, Accelerated design, Future of baccalaureate education

\section{INTRODUCTION}

Dramatic changes in healthcare, healthcare delivery, and health policy with a strong focus on the triple aim of: 1) improving the individual experience of care, 2) improving the health of populations, and 3) reducing the per capita cost of care for populations are creating new opportunities for professional registered nurses (RNs). ${ }^{[1]}$ Opportunities include participating in and leading change, and creating new roles across all healthcare settings. With an evolving focus on primary and community-based care rather than acute care, and recognition of the importance of coordinating care and managing transitions across providers and settings of care, RNs now and in the future will need to be prepared with a wide scope of knowledge, skills, and competencies. ${ }^{[2-4]}$ To prepare a healthcare workforce capable of caring for people across the lifespan from a health promotion/disease prevention construct, and a community and population health perspective, a culture change in nursing education is warranted. ${ }^{[4]}$ The purpose of this paper is to describe the structure for developing an innovative baccalaureate nursing curriculum and lessons learned that can inform the efforts of others interested in accelerating design of new curriculum.

\section{Project SCOPE}

In April 2014, the College of Nursing embarked on creating a new conceptual framework and corresponding new curricu-

*Correspondence: Mary Theresa Bouchaud, Assistant Professor; Email: Mary.Bouchaud@ jefferson.edu; Address: Jefferson College of Nursing, Thomas Jefferson University, United States. 
lum for baccalaureate nursing education. The framework and curriculum was responsive to national reports, standards, and competencies and based on the best evidence that was available to guide model creation, development, planning, implementation, and evaluation. The curriculum needed to be customer centric, and provide a quality education for students to be prepared and ready for practice.

The charge of designing an innovative baccalaureate nursing curriculum for this college of nursing was a prodigious undertaking. To begin, faculty were given 13 months, an ambitious timeframe, to accomplish designing a new BSN curriculum that entailed three pre-licensure BSN programs: 1) a second degree program for non-nurses completed over 12 months, 2) a second degree program for non-nurses completed over two academic years, and 3) an upper division traditional program completed over two academic years. The programs were to remain intact and the development and implementation of these three programs would reflect the emerging and futuristic changes in healthcare for the 21 st century. Although the expectation was that the faculty selected to champion this project would continue to teach, advise students, obtain their clinical contracts and adjunct clinical faculty, and participate in their assigned committees, the Dean did allocate one three-credit course for each of the members on the Curriculum Navigators (CNs) team to reduce their teaching workload. Other features of this faculty-driven BSN curriculum project included how the team was formed, the inclusion of a hospital RN clinical partner, a Distinguished Visiting Professor to guide the team, and the selection and inclusion process of five additional community members later known as community advisors.

\section{GetTing STARTED}

The Dean shared a summary of the project with faculty including assumptions, resources, deliverables, and an aggressive 13-month project timeline. A key beginning step for this important work was developing shared assumptions to assure that all relevant parameters for a new curricular design were addressed, including use of best evidence, attention to the student experience, consideration of resource implications, human resources, and other relevant topics. Assumptions included the University's vision to create unparalleled value by reimagining healthcare, health education, and discovery, mission that Health is all we do, and values of innovation, service excellence, collaboration, ownership, respect, and empowerment (iSCORE). Additional assumptions included a "blank slate" for changing baccalaureate nursing education, a philosophy of team teaching, student engagement, partner engagement, faculty engagement, articulation with the current Master of Science in Nursing (MSN) and Doctor of Nursing
Practice (DNP) plans of study, and a fiscally responsible approach.

Curriculum design was a faculty-led initiative that included the following activities and resources:

(1) Established a 15-member core team comprised of nine nursing faculty members, eight undergraduate and one graduate; one practice representative; one community representative; one current student; one alumni; one current clinical adjunct; and one recipient of care. A support staff was assigned to the core team.

(2) Selected two Co-chairs.

(3) Assigned core team members three credits in fall 2014 and three credits in spring 2015 for curriculum development.

(4) Engaged a Distinguished Visiting Professor who spent time in-residence during the 2014-2015 academic year, as well as provided virtual and telephonic support. She was the core team's support system and provided an external perspective, as well as guidance and expertise.

(5) Engaged additional external experts as needed, related to cultural competency, quality and safety, and clinical evaluation.

(6) Provided periodic open forums for all faculty members, and monthly updates to the faculty, Curriculum Committee, Dean, and Associate Dean.

(7) Convened a four hour kick-off meeting for all faculty members in April 2014.

(8) Provided readings prior to the kick-off meeting on Blackboard Learn accessible for all faculty members. Preliminary readings are listed in Table 1.

Table 1. Preliminary Readings

Nurse of the Future Core Competencies (Massachusetts Department of Higher Education)

Carnegie Foundation, Book Highlights from Educating Nurses: A Call for Radical Transformation (December 2009)

IOM/RWJF Report Brief with Focus on Education from The Future of Nursing: Leading Change, Advancing Health (October 2010)

National Advisory Council on Nurse Education and Practice (NACNEP), Addressing New Challenges Facing Nursing Education: Solutions for a Transforming Healthcare Environment (March 2010)

Public Health Reports, HRSA Supplement, Nursing in 3D: Workforce Diversity, Health Disparities, and Social Determinants of Health (January/February 2014)

HRSA's National Center for Health Workforce Analysis, The U.S. Nursing Workforce: Trends in Supply and Education (April 2013)

The Blue Ridge Academic Health Group, Report 18: A call to lead: The case for accelerating academic health center transformation (Spring 2014)

*Faculty were encouraged to add to this beginning collection 
The final curriculum document needed to be submitted to the Curriculum Committee for review and approval by Friday May 29, 2015. The Executive Council final review and approval was scheduled for June 24, 2015. A submission to the Pennsylvania State Board of Nursing was made on August 7, 2015. The identified deliverables were: a curriculum model, curricular themes, end-of-program curricular outcomes, student learning outcomes and level competencies, curriculum design, plan, and sequencing, competency learning progression maps, course syllabi, concept/content maps, and implementation plan.

\section{STRUCTURE FOR CURRICULUM DESIGN/DEVELOPMENT}

The experience of curricular design or change is challenging and many College nurse faculty reported prior frustration and dissatisfaction with the process and outcome (personal communication, 2015). While causal factors likely vary from school to school, there are common factors seen in many curricular projects. Factors reported by College of Nursing faculty with prior experience in curriculum redesign included: lack of knowledge regarding curriculum development; lack of consensus regarding how to organize the curriculum development work (with too much focus on details and vested interests rather than a changing external environment and stakeholder needs); the amount of time curriculum development required; and related to the prior items, concerns about approaching curriculum projects using existing faculty processes with the usual power struggles and frequently reported incivility. The authors located several studies related to developing curriculum and constructing learning environments for students, however, no studies related to curriculum development and faculty behavior were identified.

An evidence-based approach to curriculum design was introduced by the consulting visiting professor to mitigate the aforementioned commonly reported dissatisfiers. The recommended conceptual framework for curricular development included three major approaches that promoted a future orientation (idealized design); enhanced participation, decision making, and consensus building using a data driven, evidence-based approach (quality improvement principles); and, project management principles to ensure efficient and timely accomplishment of the project.

Idealized design may be defined as the process of interactive planning. ${ }^{[5]}$ There are three major steps to idealized design, namely: 1) describing the current state in relation to evolving needs based on a current scan of the external environment and stakeholders, 2) envisioning the desired future state that would achieve priority goals, and 3 ) adjusting the ideal de- sign plan to accomplish as close to the ideal as possible by modifying the vision for system limitations or other realities that likely cannot be influenced. The overall goal of idealized design is to achieve the best, creative thinking for the future rather than first being constrained by current realities. ${ }^{[5]}$

Quality improvement (QI) principles and techniques are widely used throughout the healthcare system. There are many models for QI in healthcare and other industries; yet, at their core, the majority of the principles are very similar. Important QI principles focus on customer needs, assessing and refining processes to achieve those needs using data and efficient methods, a commitment to participative strategies so that those closest to the work processes have strong voices in design and decision making, and plans for documenting improvements with a goal of continuously improving. ${ }^{[6]}$ While all of the latter are important in curriculum design projects, a change in typical faculty meeting communication and decision making activities can improve quality, efficiency, and participant satisfaction. Specifically, clarifying roles (leaders, members, facilitators, others), creating ground rules for meeting behaviors and responsibilities, articulating decision making processes, and active facilitation of precious meeting time must be enacted consistently.

Last, project management principles assure that there is a plan for sequencing and responsibilities assigned related to each phase of project activity necessary to achieve the project goals. Project management principles enhance meeting effectiveness with a shared understanding and ownership of the actions needed to move the project forward through the project lifecycle. ${ }^{[7]}$ This framework worked well as it permitted the team to dream and have a continuous feedback loop while keeping with the aggressive timeline.

\section{Core team}

Faculty interested in volunteering for the core team were asked to write a short paragraph about why they were interested in serving on the core team, what strengths they would bring to the team, and how they saw the team supporting their continued professional development. Parameters for core team composition included such characteristics as diversity of backgrounds, specialties, styles, and experiences. Twelve faculty members expressed their willingness to serve, submissions were reviewed, and selected core team members were notified. Faculty members not selected were assured that everyone would have many opportunities to contribute to the design.

Nine faculty members were selected and the Professional Development Specialist from the College of Nursing's practice partner was invited to be a member. 
Prior to the kick-off meeting, the core team sent an open letter to all undergraduate and graduate faculty members inviting their support and voice in creating a new 21 st century baccalaureate curriculum. It was critical to have meaningful faculty interactions and faculty buy-in throughout the process. The core team desired to be viewed by the faculty at large as an organizing agent for curricular change; thus, the name of Curriculum Navigators (CNs) was selected. The success of the new curriculum would be achieved through collaborative efforts and culmination of expertise by the entire faculty. The core team was committed to a process that maintained open, respectful communication, flexibility, and transparency.

The consultant engaged the full faculty in a two hour curriculum "kick off" workshop, and then met with the core team to select co-chairs. Throughout the one-year curriculum development, both co-chairs worked closely together in preparing meeting agendas, monthly meetings with the Dean, conference calls and pre-team meetings with the consultant, and preparation and implementation of all general faculty engagement workshops and presentation updates.

The initial charge from the Dean included the recruitment of five additional committee members to the core team to include: one community leader, one adjunct faculty, one current BSN student, one BSN alumnus, and one current patient care recipient. The $\mathrm{CNs}$ reviewed the 23 applications and chose five individuals to join the team. These new members were later named by the core team as "community advisors".

\section{ROLE AND EXPECTATIONS OF COMMUNITY ADVISORS}

Initially, the core team anticipated that the community advisors would attend each meeting of the $\mathrm{CNs}$ either in person or via phone conference. However, as the project progressed, the CNs soon saw the need to meet biweekly, then weekly and often held all day meetings. It soon became apparent that the community advisors were unable to keep up with this grueling schedule. Even prior to increasing the frequency of meetings, attendance and email correspondence by the advisors was sporadic, if at all, except for one who made almost every meeting. Her expertise in the corporate world and with team building and leadership proved to be invaluable to the team. As a result, the core team revised their method of gathering input from the community advisors. The advisors were asked to attend a monthly community advisor meeting dedicated to keeping them current and informed on the $\mathrm{CN}$ accomplishments and eliciting their thoughts and feedback in moving forward. They continued to be invited to all curriculum navigator meetings and to receive associated meeting minutes and agendas, and the navigator newsletters. This approach did improve, somewhat, the team's ability to obtain input and feedback from the advisors who were not regularly attending meetings. In response, the advisors expressed appreciation of being kept informed of the teams' progress and the development of the curriculum.

\section{STUDENT INVOLVEMENT AND FEEDBACK}

The final aspect of the project was involving students and soliciting their feedback. Several CNs volunteered to conduct multiple focus groups that would include graduate and undergraduate students. The intent of these focus groups was to gather data related to student perceptions of the current BSN curriculum and to elicit their suggestions, curricular themes, and overall "gut" reactions to the current curriculum and the proposed designed BSN curriculum. There were several advantages to including undergraduate and graduate nursing students to the already diverse population of individuals impacting the new BSN curriculum. To begin, there was student representation from all three undergraduate BSN programs who were at varying levels in their studies and who could speak directly to the concerns and satisfaction of classmates with the current curriculum. Secondly, including graduate students secured representation of students who have completed one of the three BSN programs, successfully passed NCLEX-RN, and are continuing on to their graduate nursing pursuits. These students bring insight related to the BSN programs they just completed and a vision for where they believe nursing student preparation should be headed.

Students participating in the focus groups were eager to participate and were engaged during the focus group sessions. They shared objective, measurable, and positive suggestions to improve the current curriculum while identifying passionately, the strengths of that curriculum including faculty delivery of classroom material, clinical sites, preparation for NCLEX-RN, and the NCLEX-RN success pass rates. In addition, after reviewing the proposed newly developed didactic and clinical immersion courses, the students from each focus group expressed their excitement for the new students who will be taking part in this new curriculum with many stating they wished this curriculum had been available when they entered the program. Student input was reviewed and incorporated into the new curriculum as applicable and appropriate. All undergraduate nursing students were kept informed of the progress of the new curriculum development via brief announcements made in their classroom courses and all were encouraged to submit any comments to the co-chairs of the CNs committee. 


\section{Faculty engagement}

Central to creating an innovative and contemporary undergraduate curriculum and paramount to the long-term success of a curriculum design, is the engagement of the entire faculty including undergraduate, graduate, adjunct faculty, and leadership. During the curriculum development process, the CNs utilized several formal and informal strategies in order to inform faculty regarding overall progress, elicit feedback based on faculty expertise, brainstorm about innovative curriculum design, and anticipate and troubleshoot future potential implementation concerns. These strategies are described in Table 2.

Table 2. Faculty Engagement Strategies

\begin{tabular}{|c|c|c|}
\hline Strategy & Description & Strength \\
\hline $\begin{array}{l}\text { Curriculum } \\
\text { Koffee Klatch }\end{array}$ & $\begin{array}{l}\text { Regular opportunity for faculty members to meet periodically } \\
\text { with the CNs during informal sessions. Faculty discussed, } \\
\text { debated, and shared ideas regarding curriculum design. }\end{array}$ & $\begin{array}{l}\text { Lack of a preplanned agenda or presentation; The feedback and } \\
\text { content of discussion was recorded and shared at the following } \\
\text { CN meeting. }\end{array}$ \\
\hline $\begin{array}{l}\text { Curriculum } \\
\text { Alerts }\end{array}$ & $\begin{array}{l}\text { Newsletter to share extensive textual information on a } \\
\text { bimonthly basis. General updates, insight regarding the } \\
\text { process of curriculum redesign, information regarding } \\
\text { opportunities for faculty to provide feedback, and recent } \\
\text { trends in nursing education. }\end{array}$ & $\begin{array}{l}\text { Vehicle for important curriculum documents to be shared with } \\
\text { the faculty in both draft and final forms. }\end{array}$ \\
\hline $\begin{array}{l}\text { Faculty } \\
\text { Workshops (2) }\end{array}$ & $\begin{array}{l}\text { Convened over a two-day period dedicated to key activities } \\
\text { that focused on needs of curriculum design. Faculty members } \\
\text { were placed in working groups based on individual expertise, } \\
\text { CN member served as a guide and scribe in order to assist the } \\
\text { group to engage in the activities. }\end{array}$ & $\begin{array}{l}\text { Explored key curriculum elements balancing idealized design } \\
\text { with quality/project management. Level competencies, course } \\
\text { syllabi, best pedagogy practices, and mapping to standards, } \\
\text { competencies, and program objectives. }\end{array}$ \\
\hline
\end{tabular}

\section{IMPLEMENTING PROJECT MANAGEMENT PROCEDURES}

Structured project management procedures ensured that the team worked effectively and efficiently. At the outset of the project, the CNs developed a working document that incorporated all milestones, project deliverables, meetings, and tasks. A list of project deliverables is included in Table 3. The first project deliverable was a list of meetings for the core team which identified dates and times for meetings through the 13month curriculum design phase. This schedule enabled the core team to understand the time commitment involved with the project while recognizing that ad hoc meetings would be needed based on project work flow. Meetings were typically three hours in length with some lasting six to eight hours depending on the agenda.

Table 3. Project Deliverables

\footnotetext{
- Curriculum framework

- Curricular themes

- Curricular outcomes for end-of program

- Student learning outcomes and level competencies

- Curriculum design, plan, and sequencing

- Competency learning progression maps

- Course syllabi

- Concept/content maps

- Implementation plan
}

Published by Sciedu Press
In addition to meetings for core team members, the CNs also established regular monthly meetings for the co-chairs and the Dean to keep her abreast of progress, resources needed, and challenges encountered. The Dean was an important source of information on regulatory and accreditation considerations related to the new curriculum.

Communication and coordination strategies were implemented to facilitate effective collaboration with the community advisors. While the community advisors were invited to attend all scheduled meetings, the CNs recognized early on that they needed to identify specific meetings in which attendance was required as competing priorities prevented their participation in every meeting. During these meetings, two to three team members reviewed work products to obtain focused feedback from the community advisors. The faculty brought the advisors' feedback to the team, discussed their feedback, and revisions were made as needed.

Because the timeline was aggressive and the CNs had parallel tasks, the use of subcommittees to accomplish work outside of regular meetings was necessary to meet target deadlines. For example, at the beginning of the design phase, the CNs conducted a review of the literature on emerging roles for registered nurses, clinical education models, inter- 
professional education, and workforce trends. Two to three team members would complete their assigned literature review and report findings to the entire team, thus, maximizing the use of meeting time. Taken together, the idealized design, quality improvement principles, and project management procedures ensured that the $\mathrm{CNs}$ produced a high-quality product on time with key stakeholder engagement throughout the process. There were many lessons learned in undertaking this complex process over a brief time period and they are described in Table 4. For example, as the scope of the project was identified and updated, it was important to continue the idealized design process as to not limit thinking or revert back to the old curriculum. Ongoing quality improvement principles ensured effective communication throughout the process - from committee communication to faculty meeting communication, to communication with volunteer stakeholders. Project management strategies supported leadership, stakeholder engagement, and changes that occurred along the way.

Table 4. Lessons Learned

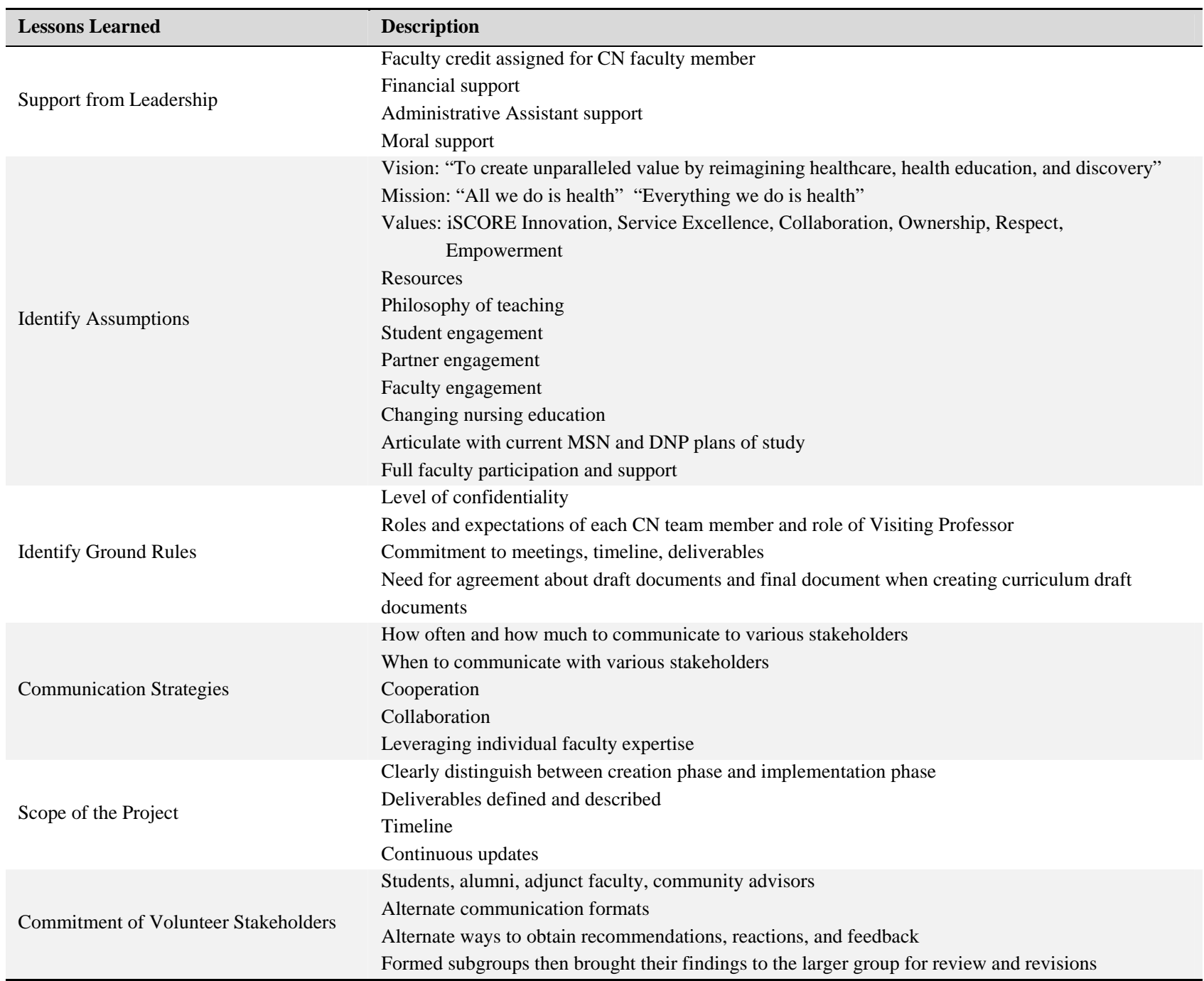

\section{THE CURRICULUM FOR HEALTH}

\section{IS H.E.R.E.}

In this context, the College of Nursing designed an innovative baccalaureate nursing curriculum based on the organization's mission, Health is All We Do and the curriculum for health is H.E.R.E. - Humanistic, Evidence-based, Reflective, and Excellence in clinical leaders(c). The curricular framework that guides the newly designed concept-based baccalaureate curriculum is Promoting Health and Quality of Life Along the Care Continuum and is depicted in Figure 1. This framework emphasizes the promotion of health and quality of life in a variety of populations during transitions of care from one setting to another and is guided by the curricular themes of innovation, population health, interprofessional collabora- 
tion, and practice excellence. The curriculum is consistent with the American Association of Colleges of Nursing's (AACN) Essentials of Baccalaureate Education for Professional Nursing Practice ${ }^{[8]}$ building upon the components of liberal arts education, professional values, core competencies, core knowledge, and role development. Central to the curriculum is the need to leverage partnerships to support the newly developed course offerings, immersion experiences (formerly clinical experiences), service learning, and experiential opportunities in interprofessional, community-based primary care. These partnerships are mutually beneficial to promote health and foster collaboration to improve wellbeing.

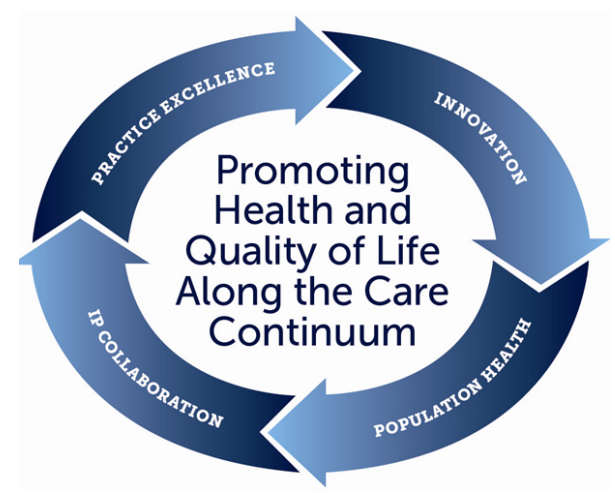

Figure 1. Curriculum Framework

The new curriculum for baccalaureate nursing students' didactic content and immersion experiences is more closely aligned with the evolving and enhanced roles of RNs beyond the hospital walls. Students engage in integrated didactic learning and immersion practicums that promote a culture of health and multiple new and emerging roles of RNs rather than a diseased-based, acute care focused curriculum. Nursing students learn content related to safe and effective primary care services delivered in community-based settings, preparing them with knowledge and skills in care coordination, chronic disease prevention, population health, and team-based, interprofessional care. Specific courses address: 1) health promotion across the lifespan, 2) professional practice, 3) discovery and evidence-based practice, 4) healthcare informatics and innovation, 5) population health, cultural awareness, and health disparities, 6) care coordination and care transitions, and 7) clinical reasoning.

\section{Summary}

Developing an innovative and futuristic baccalaureate curriculum was a journey of growth for individual faculty, as well as the faculty as a whole. The blank slate challenged faculty to be creative and fostered risk taking. Transparency played a critical role in ensuring integrity of the structure and frequent communication and dialogue facilitated full faculty participation. Diverse representation of roles, experience, expertise, and ideas supported a healthy tension and an environment for debate. The accelerated timeframe kept everyone engaged and committed. The faculty is proud that the structure to accelerate the design of an innovative baccalaureate curriculum resulted in transforming baccalaureate nursing education and fostering a culture of health as the Curriculum for Health is H.E.R.E - Humanistic, Evidence-based, Reflective, Excellent clinical leaders(C).

\section{CONFLiCTS OF INTEREST Disclosure}

The authors declare that they have no conflicts of interest.

\section{REFERENCES}

[1] Institute of Medicine. The future of nursing: Leading change, advancing health. Washington D.C. 2011.

[2] Fortier M, Fountain D, Vargas M, et al. Healthcare in the community: Developing academic/practice partnerships for care coordination and managing transitions. Nursing Economic. 2015; 33(3): 167-175, 181.

[3] Fraher E, Spetz J, Naylor M. Nursing in a transformed healthcare system: New roles, new rules. Princeton: Robert Wood Johnson Foundation. 2015.

[4] Bouchaud M. Preparing baccalaureate nursing students for community/public health nursing: Belief systems and values of nurse educators and administrators. Ann Arbor, MI: ProQuest LLC. 2011.
[5] Ackoff R, Magidson J, Addison H. Idealized design. Upper Saddle River: Prentice Hall. 2006.

[6] Beaudry M, Bialek R, Moran J. Using quality improvement tools and methods throughout the accreditation lifecycle. Journal of Public Health Management Practice. 2014; 20(1): 49-51. PMid:24322686. http://dx.doi.org/10.1097/PHH.0b013e3182a778bd

[7] Gocsik T, Barton A. Why clinical change leadership is essential for project success. Clinical Nurse Specialist. 2014; 28(2): 83 85. PMid:24504032. http://dx.doi.org/10.1097/NUR. 00000 00000000026

[8] American Association of Colleges of Nursing. The essentials of baccalaureate education for professional nursing practice. Washington D.C.: American Association of Colleges of Nursing. 2008. 